\title{
Seasonal dynamics of nitrate and ammonium ion concentrations in soil solutions collected using MacroRhizon suction cups
}

\author{
Cezary Kabala • Anna Karczewska • Bernard Gałka • \\ Mateusz Cuske • Józef Sowiński
}

Received: 16 February 2017 / Accepted: 23 May 2017 /Published online: 1 June 2017

(C) The Author(s) 2017. This article is an open access publication

\begin{abstract}
The aims of the study were to analyse the concentration of nitrate and ammonium ions in soil solutions obtained using MacroRhizon miniaturized composite suction cups under field conditions and to determine potential nitrogen leaching from soil fertilized with three types of fertilizers (standard urea, slowrelease urea, and ammonium nitrate) at the doses of 90 and $180 \mathrm{~kg} \mathrm{ha}^{-1}$, applied once or divided into two rates. During a 3-year growing experiment with sugar sorghum, the concentration of nitrate and ammonium ions in soil solutions was the highest with standard urea fertilization and the lowest in variants fertilized with slow-release urea for most of the months of the growing season. Higher concentrations of both nitrogen forms were noted at the fertilizer dose of $180 \mathrm{~kg} \mathrm{ha}^{-1}$. Onetime fertilization, at both doses, resulted in higher nitrate concentrations in June and July, while dividing the dose into two rates resulted in higher nitrate concentrations between August and November. The highest potential for nitrate leaching during the growing season was in July. The tests confirmed that the miniaturized suction cups MacroRhizon are highly useful for routine
\end{abstract}

C. Kabala $(\bowtie) \cdot$ A. Karczewska $\cdot$ B. Gałka $\cdot$ M. Cuske Institute of Soil Science and Environmental Protection, Wrocław University of Environmental and Life Sciences, ul. Norwida 25, 50-357 Wroclaw, Poland

e-mail: cezary.kabala@upwr.edu.pl

J. Sowiński

Department of Crop Production, Wrocław University of Environmental and Life Sciences, ul. Norwida 25, 50-357 Wroclaw, Poland monitoring the concentration of nitrate and ammonium ions in soil solutions under field conditions.

Keywords Nitrogen fertilization $\cdot$ Nitrates $\cdot$ Soil solution $\cdot$ Groundwater $\cdot$ Suction cups $\cdot$ Monitoring

\section{Introduction}

The environmental consequences of nitrogen fertilization are mainly related to easy nitrate leaching to the ground and surface waters that has extensive negative environmental and economic consequences (Berge et al. 2001; Csathó et al. 2007; Zakarauskaite et al. 2008). Mineral fertilizers that contain nitrogen in nitrate form present a particular threat to the waters; however, other nitrogen fertilizers, including those considered to be "safer" due to their slow decomposition rates, also undergo transformation in the soil and are a source of forms susceptible to leaching to the groundwater or to release to the atmosphere (Fotyma et al. 2010; Bouwman et al. 2013). Nitrogen transformation and leaching from arable land are complex phenomena, and the direction and intensity of these processes depend on soil properties, climate and weather conditions, and agrotechnical factors. Nitrogen losses due to leaching from soil within conventional agricultural production systems usually are in a range from $<10$ to $30 \%$ (Meisinger and Delgado 2002), although they may exceed 30\% in coarse-textured soils (Sapek 2004). European Union member states have adopted both the "Nitrate Directive" (1991/676/EC) with the aim of 
protecting waters against pollution caused by nitrates from agricultural sources and a directive (2006/118/EC) on the protection of groundwater against pollution and deterioration. Legal regulations in individual member states, including Poland (Regulation 2002), are compliant with the requirements of both these directives. The threshold concentration of nitrates $\left(\mathrm{NO}_{3}{ }^{-}\right)$in groundwater has been determined as $50 \mathrm{mg} \mathrm{dm}{ }^{-3}$, which corresponds to $11.3 \mathrm{mg} \mathrm{dm}{ }^{-3}$ of nitrate nitrogen $\left(\mathrm{N}^{-} \mathrm{NO}_{3}{ }^{-}\right)$.

The studies on nitrogen fertilization of cultivated crops must consider nitrogen losses, not only in economic terms, but also in relation to the aforementioned environmental threats. This refers in particular to coarsetextured (sandy) soils that are characterized by limited retention capacity and a poor sorption complex. Nitrogen leaching to groundwaters from such soils may be particularly intense (Hatch et al. 2010; Jadczyszyn et al. 2010).

Current concentration of nitrogen forms in soil solutions is resulting from the balance between (1) nitrogen supply with fertilizers, crop remains and dead organic matter, atmospheric deposition, assimilation of atmospheric nitrogen by bacteria and actinobacteria, and potential capillary rise; (2) losses caused by plant uptake, release to the atmosphere in gaseous form and leaching to waters; and (3) transformations of certain forms of nitrogen into other ones in course of mineralisation, ammonification, nitrification, and denitrification processes (Georgallas et al. 2012). Both the uptake, transformation, and leaching/release of particular nitrogen forms are influenced by temperature and moisture (Glina et al. 2016). Due to the complex nature of all these processes, the concentrations of nitrate and ammonium ions in soil solutions are characterized by high levels of variability and dynamics (Spohn et al. 2016).

The descending water movement through the soil profile under the temperate climate (as in Central Europe), especially in sandy soils, may result in a close correlation between high concentrations of mineral nitrogen forms in soil solution of plough layer and their high concentrations in a shallow groundwater and, as a consequence, also in deeper groundwater and in surface waters. Therefore, the monitoring of soil solution may contribute to an explanation of the relationships between nutrient uptake by plants and leaching to groundwater (Jadczyszyn et al. 2010). However, direct monitoring of the concentrations of nitrogen forms in soil solutions involves a series of technical problems. Fortunately, many of them have been solved with the introduction of ceramic suction cups (Webster et al. 1993).
Conventional ceramic samplers are, however, expensive and require a pump to create underpressure, which greatly increases the costs of installation and limits the number of objects monitored at the same time, thus precluding the application of this measurement method from multi-site projects, as for example the multi-plot agricultural field experiments. Miniaturized composite suction cups are significantly cheaper than ceramic ones, and they require a simple plastic syringe instead of a pump to create underpressure. Low costs of installation and operating allow mass application of the miniaturized suction cups in the monitoring of soil solution dynamics. The aim of the investigation was to analyse the seasonal dynamics of the concentration of ammonium and nitrate ions in the soil solutions obtained with use of miniaturized suction cups MacroRhizon in a multi-plot standard fertilization experiment on sandy soil to assess the usefulness of such type of suction cups in the monitoring of the threats for a shallow groundwater quality.

\section{Materials and methods}

An experiment with sweet sorghum cultivated for biomass aimed at food and energy production was conducted in years 2013-2015 at the experimental field station of the Department of Crop Production of Wroclaw University of Environmental and Life Sciences. According to WRB classification (Kabala et al. 2016a), the experiment was conducted on sandy-textured soils, originally Brunic Arenosols, recently converted into Gleyic Phaeozems (Anthric, Arenic, Brunic) due to long-term and intense cultivation, including deep ploughing, liming, and fertilization (Labaz and Kabala 2016). The soils have a thick humus horizon (28$32 \mathrm{~cm}$ ), characterized by medium-high content of organic carbon $(0.5-2 \%)$, neutral reaction, and high base saturation $(>75 \%)$. A detailed description of the physico-chemical soil properties and the content of mineral forms of nitrogen in the bulk soil have been presented separately (Gałka et al. 2016; Kabala et al. 2016b; Sowiński et al. 2016).

The experiment was conducted on 52 plots using the random block method, and the analysed variance factors were (1) the fertilizer type (ammonium nitrate, standard urea, and slow-release (coated) urea); (2) fertilizer dose (90 and $180 \mathrm{~kg} \mathrm{ha}^{-1}$ ); and (3) application strategy (whole dose in one rate or dose divided into two rates). 
Nitrogen fertilization (whole dose or the first half of the dose) was applied at the end of May (two-three-leaf phase), while the second half of the dose (if divided) was applied in mid-July.

The total precipitation during the growing season was strongly differentiated in the subsequent years (Table 1). In 2013, the total precipitation in the period April-October was $233.4 \mathrm{~mm}$ higher than the long-term average. In 2014, the precipitation was distributed more evenly, and the total rainfall in the period April-October was $96.8 \mathrm{~mm}$ higher than average, whereas the year 2015 was characterized by a strong water deficit. Total precipitation was only $191.3 \mathrm{~mm}$, which was $168.2 \mathrm{~mm}$ lower than the long-term average for the growing season. In 2013 and 2014, the mean air temperature during the sorghum vegetation season was higher than longterm average, by 0.5 and $0.6{ }^{\circ} \mathrm{C}$, respectively, while in 2015 , it remained similar to the average (Table 1).

On each experimental plot, at the depth of approx. $25 \mathrm{~cm}$ (in the bottom part of the humus layer) and approx. $50 \mathrm{~cm}$ (below plough layer), MacroRhizon composite suction cups (Rhizosphere Research Products, Wageningen, The Netherlands) were installed in two replicates (at each depth). The porous section of the cups was $90 \mathrm{~mm}$ long and $4.5 \mathrm{~mm}$ thick (the external diameter). Previous research had demonstrated that MacroRhizon suction cups are characterized by a minimal own sorption of anionic and cationic components, which makes them suitable for analysing natural soil solutions (Cuske et al. 2017; Hatch et al. 2010; Jämtgård et al. 2010; Kabala et al. 2014). Another advantage is their low price in comparison to ceramic, glass, or silicone samplers. This type of sampler may be used in the underpressure range 20-50 $\mathrm{kPa}$ (Iost et al. 2012).

Suction cups were installed in soil in an inclined position (at an angle of approx. $45^{\circ}$ ). Continuous contact of the sampler surface with soil was ensured by sealing them with a pulp of fine-grained sand. The ends of discharging silicone pipes and the collecting
$P E$ vessels were placed in subsurface wells made of PVC pipes of a diameter of $11 \mathrm{~cm}$ and a depth of $50 \mathrm{~cm}$. Soil solution samples were collected actively, by applying negative pressure generated by a syringe piston of a volume of $50 \mathrm{~cm}^{3}$. The solutions were collected throughout the growing season, i.e. from May to November. Initially, it was planned to collect soil solution samples at regular 10-day intervals. However, long periods without precipitation often made impossible either to collect the required volume or to collect any solutions for analysis from all experimental plots in a very regular interval. Thus, solution samples were collected at 7-24-day intervals, at a sufficient soil moisture. Consequently, the current summary presents the concentrations of nitrogen forms averaged for months. Due to the limited possibility to collect soil solutions in winter and due to the necessity to cultivate soil on the experimental plots, the suction cups and wells were removed before winter (in December) and re-installed in the following spring.

The collected soil solution samples were cooled and transported to the laboratory immediately. After filtration, the concentrations of nitrate and ammonium ions were determined. The concentration of $\mathrm{NO}_{3}$ ions was determined with the potentiometric method with the use of an ionic selective nitrate electrode (Dojlido and Zerbe 1997). The concentration of ammonium ions in soil solutions was determined with the colorimetric method, using Nessler reagent (Siepak 1992). The concentrations of $\mathrm{NO}_{2}{ }^{-}$ions in soil solutions were not determined routinely, as they had initially been estimated as being of negligibly low concentrations (by the colorimetric method, Spectroquant tests, Merck Millipore). The statistical analysis of the results was performed with use of STATISTICA 10 software, ANOVA/MANOVA package, to evaluate the influence of fertilizer, dose, and application strategy and the interactions between the analysed factors. Significance was calculated at $p<0.05$.

Table 1 Mean sum of precipitation and mean air temperature during growing periods (April to October) in years 2013-2015

\begin{tabular}{lrrrr}
\hline & \multicolumn{2}{l}{ Year } & & Multiyear average \\
\cline { 2 - 5 } & 2013 & 2014 & 2015 & \\
\hline Sum of precipitation $(\mathrm{mm})$ & 568.4 & 456.3 & 191.3 & 359.5 \\
Mean air temperature $\left({ }^{\circ} \mathrm{C}\right)$ & 14.9 & 15.0 & 15.1 & 14.4 \\
\hline
\end{tabular}

Data from local weather station situated in the experimental station in Wrocław-Pawłowice 


\section{Results and discussion}

The $\mathrm{N}-\mathrm{NO}_{3}$ and $\mathrm{N}-\mathrm{NH}_{4}$ concentrations in the soil solution were highly variable, in both the topsoil (Tables 2 and 3 ) and subsoil (Tables 4 and 5) layers. The extremely high monthly fluctuations, particularly of nitrates, make it unreasonable to generate average results for the whole year, so statistical calculations were performed for average monthly results only.
The highest $\mathrm{N}^{-\mathrm{NO}_{3}}$ concentrations in soil solutions (averaged monthly) were noted in July 2014, up to $882 \mathrm{mg} \mathrm{dm}^{-3}$ in the topsoil (Table 2) and $102 \mathrm{mg} \mathrm{dm}^{-3}$ in the subsoil layer (Table 4). In the other years, the highest nitrate concentrations were also noted in July, resulting in the highest mean concentration across the growing season (Fig. 1). In subsequent summer months, the concentration of $\mathrm{N}-\mathrm{NO}_{3}$ in topsoil soil solution decreased to $2.16 \mathrm{mg} \mathrm{dm}^{-3}$ (in August),

Table 2 Concentration of nitrate nitrogen $\left(\mathrm{N}-\mathrm{NO}_{3}\right)$ in soil solution at the depth of $25 \mathrm{~cm}$ (topsoil layer) in years $2013-2015$ (mean values)

\begin{tabular}{|c|c|c|c|c|c|c|c|c|}
\hline \multirow[t]{2}{*}{ Fertilizer } & \multirow{2}{*}{$\begin{array}{l}\text { Rate } \\
\left(\mathrm{kg} \mathrm{ha}^{-1}\right)\end{array}$} & \multirow{2}{*}{$\begin{array}{l}\text { Dose } \\
\text { dividing }\end{array}$} & \multicolumn{6}{|l|}{ Month } \\
\hline & & & $\begin{array}{l}\text { Jun } \\
\left(\mathrm{mg} \mathrm{dm}^{-3}\right)\end{array}$ & $\begin{array}{l}\text { Jul } \\
\left(\mathrm{mg} \mathrm{dm}^{-3}\right)\end{array}$ & $\begin{array}{l}\text { Aug } \\
\left(\mathrm{mg} \mathrm{dm}^{-3}\right)\end{array}$ & $\begin{array}{l}\text { Sep } \\
\left(\mathrm{mg} \mathrm{dm}^{-3}\right)\end{array}$ & $\begin{array}{l}\text { Oct } \\
\left(\mathrm{mg} \mathrm{dm}^{-3}\right)\end{array}$ & $\begin{array}{l}\text { Nov } \\
\left(\mathrm{mg} \mathrm{dm}^{-3}\right)\end{array}$ \\
\hline \multicolumn{3}{|l|}{ Control } & 23.1 & 85.5 & 1.81 & 0.49 & 0.46 & 0.91 \\
\hline \multirow[t]{4}{*}{ Ammonium nitrate } & 90 & $1 \times$ & 51.1 & 480 & 0.99 & 0.55 & 0.53 & 1.90 \\
\hline & 90 & $2 \times$ & 40.0 & 110 & 2.05 & 0.72 & 0.47 & 2.17 \\
\hline & 180 & $1 \times$ & 63.8 & 683 & 2.31 & 0.55 & 1.23 & 1.94 \\
\hline & 180 & $2 \times$ & 39.0 & 121 & 4.64 & 1.93 & 2.47 & 3.30 \\
\hline \multirow[t]{4}{*}{ Standard urea } & 90 & $1 \times$ & 45.5 & 530 & 0.75 & 0.41 & 0.50 & 1.59 \\
\hline & 90 & $2 \times$ & 22.6 & 104 & 1.17 & 2.18 & 0.40 & 1.81 \\
\hline & 180 & $1 \times$ & 34.6 & 887 & 3.64 & 1.09 & 1.59 & 2.23 \\
\hline & 180 & $2 \times$ & 36.4 & 178 & 3.91 & 2.52 & 1.23 & 3.35 \\
\hline \multirow[t]{4}{*}{ Coated urea } & 90 & $1 \times$ & 31.6 & 468 & 1.06 & 0.91 & 0.33 & 1.34 \\
\hline & 90 & $2 \times$ & 33.8 & 88.7 & 1.08 & 0.36 & 0.66 & 1.57 \\
\hline & 180 & $1 \times$ & 33.3 & 601 & 1.93 & 1.09 & 0.52 & 1.87 \\
\hline & 180 & $2 \times$ & 27.7 & 138 & 2.39 & 1.27 & 0.90 & 1.94 \\
\hline \multicolumn{9}{|l|}{ Average for fertilizer } \\
\hline Ammonium nitrate & - & - & $48.5 \mathrm{a}$ & $348 \mathrm{a}$ & $2.49 \mathrm{a}$ & $0.94 \mathrm{a}$ & $1.17 \mathrm{a}$ & $2.33 \mathrm{a}$ \\
\hline Standard urea & - & - & $34.8 \mathrm{~b}$ & $425 \mathrm{~b}$ & $2.37 \mathrm{a}$ & $1.55 \mathrm{a}$ & $0.93 \mathrm{ab}$ & $2.24 \mathrm{a}$ \\
\hline Coated urea & - & - & $31.6 \mathrm{~b}$ & $324 \mathrm{a}$ & $1.61 \mathrm{a}$ & $0.91 \mathrm{a}$ & $0.60 \mathrm{~b}$ & $1.68 \mathrm{a}$ \\
\hline \multicolumn{9}{|c|}{ Average for fertilizer rate } \\
\hline- & 0 & - & $23.1 \mathrm{a}$ & $85.5 \mathrm{a}$ & $1.81 \mathrm{~b}$ & $0.49 \mathrm{a}$ & $0.46 \mathrm{a}$ & $0.91 \mathrm{a}$ \\
\hline- & 90 & - & $36.9 \mathrm{~b}$ & $273 \mathrm{~b}$ & $1.18 \mathrm{a}$ & $0.85 \mathrm{~b}$ & $0.50 \mathrm{a}$ & $1.72 \mathrm{~b}$ \\
\hline- & 180 & - & $39.1 \mathrm{~b}$ & $435 \mathrm{c}$ & $3.13 \mathrm{c}$ & $1.14 \mathrm{~b}$ & $1.32 \mathrm{~b}$ & $2.44 \mathrm{~b}$ \\
\hline \multicolumn{9}{|c|}{ Average for dose dividing } \\
\hline- & - & $1 \times$ & $43.3 \mathrm{a}$ & $608 \mathrm{a}$ & $1.78 \mathrm{a}$ & $0.76 \mathrm{a}$ & $0.78 \mathrm{a}$ & $1.81 \mathrm{a}$ \\
\hline- & - & $2 \times$ & $33.3 \mathrm{a}$ & $123 \mathrm{~b}$ & $2.54 \mathrm{a}$ & $1.50 \mathrm{~b}$ & $1.02 \mathrm{a}$ & $2.35 \mathrm{a}$ \\
\hline \multicolumn{9}{|l|}{ Average for years } \\
\hline 2013 & & & $52.5 \mathrm{a}$ & $144 \mathrm{a}$ & $2.96 \mathrm{a}$ & $1.33 \mathrm{a}$ & $0.90 \mathrm{a}$ & $1.62 \mathrm{a}$ \\
\hline 2014 & & & $23.7 \mathrm{~b}$ & $882 \mathrm{~b}$ & $1.35 \mathrm{~b}$ & $0.93 \mathrm{a}$ & $0.95 \mathrm{a}$ & $3.02 \mathrm{~b}$ \\
\hline 2015 & & & $37.8 \mathrm{ab}$ & $71.1 \mathrm{a}$ & - & - & $0.86 \mathrm{a}$ & $1.61 \mathrm{a}$ \\
\hline
\end{tabular}

Letters $\mathrm{a}$ and $\mathrm{b}$ : homogeneous groups according to the Duncan test, at $p<0.05$. The test was calculated separately for each month and independently for each factor (fertilizer type, fertilizer rate, dose dividing, year of experiment). The same letter indicates no difference between means for a particular factor in a particular month 
Table 3 Concentration of ammonium nitrogen $\left(\mathrm{N}-\mathrm{NH}_{4}\right)$ in soil solution at the depth of $25 \mathrm{~cm}$ (topsoil layer) in years $2013-2015$ (mean values)

\begin{tabular}{|c|c|c|c|c|c|c|c|c|}
\hline \multirow[t]{2}{*}{ Fertilizer } & \multirow{2}{*}{$\begin{array}{l}\text { Rate } \\
\left(\mathrm{kg} \mathrm{ha}^{-1}\right)\end{array}$} & \multirow{2}{*}{$\begin{array}{l}\text { Dose } \\
\text { dividing }\end{array}$} & \multicolumn{6}{|l|}{ Month } \\
\hline & & & $\begin{array}{l}\text { Jun } \\
\left(\mathrm{mg} \mathrm{dm}^{-3}\right)\end{array}$ & $\begin{array}{l}\text { Jul } \\
\left(\mathrm{mg} \mathrm{dm}^{-3}\right)\end{array}$ & $\begin{array}{l}\text { Aug } \\
\left(\mathrm{mg} \mathrm{dm}^{-3}\right)\end{array}$ & $\begin{array}{l}\text { Sep } \\
\left(\mathrm{mg} \mathrm{dm}^{-3}\right)\end{array}$ & $\begin{array}{l}\text { Oct } \\
\left.(\mathrm{mg} \mathrm{dm})^{-3}\right)\end{array}$ & $\begin{array}{l}\text { Nov } \\
\left(\mathrm{mg} \mathrm{dm}^{-3}\right)\end{array}$ \\
\hline \multicolumn{3}{|l|}{ Control } & 0.27 & 0.90 & 0.56 & 0.43 & 0.28 & 0.10 \\
\hline \multirow[t]{4}{*}{ Ammonium nitrate } & 90 & $1 \times$ & 0.40 & 1.17 & 1.07 & 0.90 & 0.33 & 0.51 \\
\hline & 90 & $2 \times$ & 0.40 & 1.11 & 0.84 & 0.87 & 0.57 & 0.56 \\
\hline & 180 & $1 \times$ & 0.46 & 1.90 & 2.19 & 1.22 & 0.34 & 0.42 \\
\hline & 180 & $2 \times$ & 0.66 & 1.84 & 1.76 & 1.96 & 0.63 & 0.59 \\
\hline \multirow[t]{4}{*}{ Standard urea } & 90 & $1 \times$ & 0.91 & 1.35 & 0.89 & 1.26 & 0.34 & 0.25 \\
\hline & 90 & $2 \times$ & 0.72 & 1.28 & 2.06 & 0.91 & 0.58 & 0.47 \\
\hline & 180 & $1 \times$ & 0.68 & 1.85 & 2.42 & 1.89 & 0.73 & 0.36 \\
\hline & 180 & $2 \times$ & 0.75 & 1.50 & 2.78 & 1.56 & 0.94 & 0.73 \\
\hline \multirow[t]{4}{*}{ Coated urea } & 90 & $1 \times$ & 0.47 & 1.66 & 0.71 & 0.88 & 0.35 & 0.12 \\
\hline & 90 & $2 \times$ & 0.48 & 1.36 & 0.86 & 0.74 & 0.56 & 0.20 \\
\hline & 180 & $1 \times$ & 0.56 & 1.25 & 0.91 & 1.51 & 0.60 & 0.25 \\
\hline & 180 & $2 \times$ & 0.51 & 1.30 & 1.09 & 1.26 & 0.73 & 0.38 \\
\hline \multicolumn{9}{|l|}{ Average for fertilizer } \\
\hline Ammonium nitrate & - & - & $0.48 \mathrm{a}$ & $1.50 \mathrm{a}$ & $1.46 \mathrm{~b}$ & $1.24 \mathrm{a}$ & $0.47 \mathrm{a}$ & $0.52 \mathrm{~b}$ \\
\hline Standard urea & - & - & $0.77 \mathrm{~b}$ & $1.50 \mathrm{a}$ & $2.04 \mathrm{c}$ & $1.40 \mathrm{a}$ & $0.65 \mathrm{a}$ & $0.45 \mathrm{~b}$ \\
\hline Coated urea & - & - & $0.50 \mathrm{a}$ & $1.40 \mathrm{a}$ & $0.89 \mathrm{a}$ & $1.10 \mathrm{a}$ & $0.56 \mathrm{a}$ & $0.24 \mathrm{a}$ \\
\hline \multicolumn{9}{|c|}{ Average for fertilizer rate } \\
\hline- & 0 & - & $0.27 \mathrm{a}$ & $0.90 \mathrm{a}$ & $0.56 \mathrm{a}$ & $0.43 \mathrm{a}$ & $0.28 \mathrm{a}$ & $0.10 \mathrm{a}$ \\
\hline- & 90 & - & $0.58 \mathrm{~b}$ & $1.29 \mathrm{~b}$ & $1.07 \mathrm{~b}$ & $0.93 \mathrm{~b}$ & $0.44 \mathrm{~b}$ & $0.35 \mathrm{~b}$ \\
\hline- & 180 & - & $0.60 \mathrm{~b}$ & $1.61 \mathrm{c}$ & $1.86 \mathrm{c}$ & $1.56 \mathrm{c}$ & $0.66 \mathrm{c}$ & $0.45 \mathrm{~b}$ \\
\hline \multicolumn{9}{|c|}{ Average for dose dividing } \\
\hline- & - & $1 \times$ & $0.58 \mathrm{a}$ & $1.53 \mathrm{a}$ & $1.36 \mathrm{a}$ & $1.28 \mathrm{a}$ & $0.45 \mathrm{a}$ & $0.32 \mathrm{a}$ \\
\hline- & - & $2 \times$ & $0.59 \mathrm{a}$ & $1.40 \mathrm{a}$ & $1.56 \mathrm{a}$ & $1.22 \mathrm{a}$ & $0.67 \mathrm{a}$ & $0.49 \mathrm{a}$ \\
\hline \multicolumn{9}{|l|}{ Average for years } \\
\hline 2013 & & & $0.41 \mathrm{a}$ & $1.56 \mathrm{ab}$ & $1.00 \mathrm{a}$ & $1.21 \mathrm{a}$ & $0.72 \mathrm{~b}$ & $0.35 \mathrm{a}$ \\
\hline 2014 & & & $0.60 \mathrm{ab}$ & $1.91 \mathrm{~b}$ & $1.93 \mathrm{~b}$ & $1.29 \mathrm{a}$ & $0.58 \mathrm{ab}$ & $0.56 \mathrm{a}$ \\
\hline 2015 & & & $0.73 \mathrm{~b}$ & $0.94 \mathrm{a}$ & - & - & $0.38 \mathrm{a}$ & $0.30 \mathrm{a}$ \\
\hline
\end{tabular}

Letters a and b: homogeneous groups according to the Duncan test, at $p<0.05$. The test was calculated separately for each month and independently for each factor (fertilizer type, fertilizer rate, dose dividing, year of experiment). The same letter indicates no difference between means for a particular factor in a particular month.

dropped to a minimum of $0.90 \mathrm{mg} \mathrm{dm}^{-3}$ in October, and then increased significantly in November (Fig. 1, Table 6). The concentrations of $\mathrm{N}_{-} \mathrm{NO}_{3}$ in the subsoil layer were characterized by similar seasonal fluctuations as in the topsoil, but on a much lower scale (Fig. 2).

Extremely high fluctuations of the nitrate concentrations in the soil solution during the vegetation period have also been described by other investigators, including Kroes and Roelsma (2007). The maximum concentrations of nitrates in July may result either from the application of nitrogen fertilization in the preceding weeks, as stated by Perego et al. (2012) and Sapek (2004), or from the high temperatures of the air and soil that foster fast mineralisation of organic matter from crop remains or organic fertilization in the preceding years (Georgallas et al. 2012; Sierra et al. 2015; Sapek 2010). Fluctuation of nitrate concentrations in the soil solutions in the control plots (not fertilized with nitrogen during the test period) was only slightly lower than in the fertilized plots that provides strong argument 
Table 4 Concentration of nitrate nitrogen $\left(\mathrm{N}-\mathrm{NO}_{3}\right)$ in soil solution at the depth of $50 \mathrm{~cm}$ (subsoil layer) in years $2013-2015$ (mean values)

\begin{tabular}{|c|c|c|c|c|c|c|c|c|}
\hline \multirow[t]{2}{*}{ Fertilizer } & \multirow{2}{*}{$\begin{array}{l}\text { Rate } \\
\left(\mathrm{kg} \mathrm{ha}^{-1}\right)\end{array}$} & \multirow{2}{*}{$\begin{array}{l}\text { Dose } \\
\text { dividing }\end{array}$} & \multicolumn{6}{|l|}{ Month } \\
\hline & & & $\begin{array}{l}\text { Jun } \\
\left(\mathrm{mg} \mathrm{dm}^{-3}\right)\end{array}$ & $\begin{array}{l}\text { Jul } \\
\left(\mathrm{mg} \mathrm{dm}^{-3}\right)\end{array}$ & $\begin{array}{l}\text { Aug } \\
\left(\mathrm{mg} \mathrm{dm}^{-3}\right)\end{array}$ & $\begin{array}{l}\text { Sep } \\
\left(\mathrm{mg} \mathrm{dm}^{-3}\right)\end{array}$ & $\begin{array}{l}\text { Oct } \\
\left(\mathrm{mg} \mathrm{dm}^{-3}\right)\end{array}$ & $\begin{array}{l}\text { Nov } \\
\left(\mathrm{mg} \mathrm{dm}^{-3}\right)\end{array}$ \\
\hline \multirow{5}{*}{$\begin{array}{l}\text { Control } \\
\text { Ammonium } \\
\text { nitrate }\end{array}$} & & & 16.7 & 47.0 & 0.69 & 0.38 & 0.37 & 0.59 \\
\hline & 90 & $1 \times$ & 24.7 & 62.2 & 0.52 & 0.43 & 0.43 & 0.68 \\
\hline & 90 & $2 \times$ & 21.8 & 38.9 & 0.48 & 0.55 & 0.42 & 0.62 \\
\hline & 180 & $1 \times$ & 28.0 & 77.4 & 1.63 & 0.35 & 0.83 & 0.91 \\
\hline & 180 & $2 \times$ & 25.0 & 62.2 & 1.03 & 0.31 & 0.68 & 0.84 \\
\hline \multirow[t]{4}{*}{ Standard urea } & 90 & $1 \times$ & 22.9 & 56.6 & 1.14 & 0.47 & 0.59 & 0.66 \\
\hline & 90 & $2 \times$ & 16.0 & 53.3 & 0.53 & 0.50 & 0.33 & 0.68 \\
\hline & 180 & $1 \times$ & 25.3 & 121 & 4.87 & 0.57 & 1.01 & 1.00 \\
\hline & 180 & $2 \times$ & 22.6 & 77.3 & 0.50 & 0.51 & 0.51 & 0.74 \\
\hline \multirow[t]{4}{*}{ Coated urea } & 90 & $1 \times$ & 23.1 & 55.6 & 1.02 & 0.61 & 0.59 & 0.49 \\
\hline & 90 & $2 \times$ & 18.7 & 43.0 & 0.58 & 0.18 & 0.30 & 0.65 \\
\hline & 180 & $1 \times$ & 22.6 & 80.1 & 1.46 & 0.72 & 0.72 & 0.73 \\
\hline & 180 & $2 \times$ & 16.3 & 65.5 & 0.76 & 0.47 & 0.56 & 0.79 \\
\hline \multicolumn{9}{|c|}{ Average for fertilizer } \\
\hline $\begin{array}{l}\text { Ammonium } \\
\text { nitrate }\end{array}$ & - & - & $24.9 \mathrm{~b}$ & $60.2 \mathrm{a}$ & $0.91 \mathrm{a}$ & $0.41 \mathrm{a}$ & $0.59 \mathrm{a}$ & $0.76 \mathrm{a}$ \\
\hline Standard urea & - & - & $21.7 \mathrm{a}$ & $77.1 \mathrm{a}$ & $1.76 \mathrm{~b}$ & $0.51 \mathrm{a}$ & $0.61 \mathrm{a}$ & $0.77 \mathrm{a}$ \\
\hline Coated urea & - & - & $20.2 \mathrm{a}$ & $61.0 \mathrm{a}$ & $0.95 \mathrm{a}$ & $0.49 \mathrm{a}$ & $0.54 \mathrm{a}$ & $0.66 \mathrm{a}$ \\
\hline \multicolumn{9}{|c|}{ Average for fertilizer rate } \\
\hline- & 0 & - & $16.7 \mathrm{a}$ & $47.0 \mathrm{a}$ & $0.69 \mathrm{a}$ & $0.38 \mathrm{a}$ & $0.37 \mathrm{a}$ & $0.59 \mathrm{a}$ \\
\hline- & 90 & - & $20.9 \mathrm{~b}$ & $49.2 \mathrm{a}$ & $0.71 \mathrm{a}$ & $0.45 \mathrm{a}$ & $0.44 \mathrm{a}$ & $0.62 \mathrm{a}$ \\
\hline- & 180 & - & $23.3 \mathrm{~b}$ & $80.6 \mathrm{~b}$ & $1.71 \mathrm{~b}$ & $0.49 \mathrm{a}$ & $0.72 \mathrm{~b}$ & $0.84 \mathrm{~b}$ \\
\hline \multicolumn{9}{|c|}{ Average for dose dividing } \\
\hline- & - & $1 \times$ & $24.4 \mathrm{~b}$ & $75.5 \mathrm{a}$ & $1.77 \mathrm{~b}$ & $0.52 \mathrm{a}$ & $0.69 \mathrm{a}$ & $0.74 \mathrm{a}$ \\
\hline- & - & $2 \times$ & $20.1 \mathrm{a}$ & $56.7 \mathrm{a}$ & $0.65 \mathrm{a}$ & $0.42 \mathrm{a}$ & $0.47 \mathrm{a}$ & $0.72 \mathrm{a}$ \\
\hline \multicolumn{9}{|l|}{ Average for years } \\
\hline 2013 & & & $27.7 \mathrm{a}$ & $68.7 \mathrm{ab}$ & $2.17 \mathrm{~b}$ & $0.82 \mathrm{~b}$ & $1.02 \mathrm{~b}$ & $0.91 \mathrm{~b}$ \\
\hline 2014 & & & $20.3 \mathrm{a}$ & $102 \mathrm{~b}$ & $0.24 \mathrm{a}$ & $0.13 \mathrm{a}$ & $0.26 \mathrm{a}$ & $0.59 \mathrm{a}$ \\
\hline 2015 & & & $18.7 \mathrm{a}$ & $27.9 \mathrm{a}$ & - & - & $0.47 \mathrm{a}$ & $0.70 \mathrm{ab}$ \\
\hline
\end{tabular}

Letters a and b: homogeneous groups according to the Duncan test, at $p<0.05$. The test was calculated separately for each month and independently for each factor (fertilizer type, fertilizer rate, dose dividing, year of experiment). The same letter indicates no difference between means for a particular factor in a particular month.

supporting the primary influence of weather conditions (mainly air temperature) on the concentration of nitrate ions in the soil solutions. The experiment also confirmed the late-autumn increase in the concentration of nitrates, usually linked to preceding periods of summer droughts (Trindade et al. 1997) or to decreased absorption of nitrates by agricultural crops (Gabriel et al. 2012). Increases in the concentrations of nitrates in soil solutions combined with the absence of vegetation cover and decreased microbiological activity in soils in the winter period may result in increased nitrate leaching into groundwaters (Duer et al. 2002; Sapek 2004).

Nitrate concentrations in water that exceed $50 \mathrm{mg} \mathrm{dm}^{-3}$ (equivalent to $11.3 \mathrm{mg} \mathrm{N}-\mathrm{NO}_{3} \mathrm{dm}^{-3}$ ) are considered excessive due to their risk to human health (EC Directive 2006/118/EC). The specified limit may refer to soil solution only to a certain extent. Although the soil solution directly supplies the groundwater, a significant proportion of the ions in the topsoil layer is absorbed from the solution by plant roots and 
Table 5 Concentration of ammonium nitrogen $\left(\mathrm{N}-\mathrm{NH}_{4}\right)$ in soil solution at the depth of $50 \mathrm{~cm}$ (subsoil layer) in years $2013-2015(\mathrm{mean}$ values)

\begin{tabular}{|c|c|c|c|c|c|c|c|c|}
\hline \multirow[t]{2}{*}{ Fertilizer } & \multirow{2}{*}{$\begin{array}{l}\text { Rate } \\
\left(\mathrm{kg} \mathrm{ha}^{-1}\right)\end{array}$} & \multirow{2}{*}{$\begin{array}{l}\text { Dose } \\
\text { dividing }\end{array}$} & \multicolumn{6}{|l|}{ Month } \\
\hline & & & $\begin{array}{l}\text { Jun } \\
\left(\mathrm{mg} \mathrm{dm}^{-3}\right)\end{array}$ & $\begin{array}{l}\text { Jul } \\
\left(\mathrm{mg} \mathrm{dm}^{-3}\right)\end{array}$ & $\begin{array}{l}\text { Aug } \\
\left(\mathrm{mg} \mathrm{dm}^{-3}\right)\end{array}$ & $\begin{array}{l}\text { Sep } \\
\left(\mathrm{mg} \mathrm{dm}{ }^{-3}\right)\end{array}$ & $\begin{array}{l}\text { Oct } \\
\left(\mathrm{mg} \mathrm{dm}^{-3}\right)\end{array}$ & $\begin{array}{l}\text { Nov } \\
\left(\mathrm{mg} \mathrm{dm}^{-3}\right)\end{array}$ \\
\hline \multicolumn{3}{|l|}{ Control } & 0.17 & 0.76 & 0.40 & 0.33 & 0.30 & 0.09 \\
\hline \multirow{4}{*}{$\begin{array}{l}\text { Ammonium } \\
\text { nitrate }\end{array}$} & 90 & $1 \times$ & 0.30 & 0.88 & 0.62 & 0.46 & 0.38 & 0.13 \\
\hline & 90 & $2 \times$ & 0.24 & 0.67 & 0.37 & 0.35 & 0.42 & 0.13 \\
\hline & 180 & $1 \times$ & 0.45 & 1.61 & 0.62 & 0.64 & 0.55 & 0.17 \\
\hline & 180 & $2 \times$ & 0.37 & 1.25 & 0.40 & 0.48 & 0.44 & 0.16 \\
\hline \multirow[t]{4}{*}{ Standard urea } & 90 & $1 \times$ & 0.32 & 0.92 & 0.42 & 0.51 & 0.41 & 0.16 \\
\hline & 90 & $2 \times$ & 0.23 & 0.66 & 0.38 & 0.44 & 0.33 & 0.14 \\
\hline & 180 & $1 \times$ & 0.45 & 1.49 & 0.58 & 0.69 & 0.68 & 0.25 \\
\hline & 180 & $2 \times$ & 0.37 & 1.29 & 0.45 & 0.55 & 0.58 & 0.20 \\
\hline \multirow[t]{4}{*}{ Coated urea } & 90 & $1 \times$ & 0.23 & 0.93 & 0.51 & 0.51 & 0.46 & 0.14 \\
\hline & 90 & $2 \times$ & 0.17 & 0.64 & 0.49 & 0.46 & 0.36 & 0.13 \\
\hline & 180 & $1 \times$ & 0.28 & 1.43 & 0.73 & 0.63 & 0.55 & 0.19 \\
\hline & 180 & $2 \times$ & 0.28 & 1.25 & 0.43 & 0.48 & 0.46 & 0.15 \\
\hline \multicolumn{9}{|c|}{ Average for fertilizer } \\
\hline $\begin{array}{l}\text { Ammonium } \\
\text { nitrate }\end{array}$ & - & - & $0.34 \mathrm{~b}$ & $1.10 \mathrm{a}$ & $0.50 \mathrm{a}$ & $0.48 \mathrm{a}$ & $0.45 \mathrm{a}$ & $0.15 \mathrm{a}$ \\
\hline Standard urea & - & - & $0.34 \mathrm{~b}$ & $1.09 \mathrm{a}$ & $0.45 \mathrm{a}$ & $0.55 \mathrm{a}$ & $0.50 \mathrm{a}$ & $0.19 \mathrm{a}$ \\
\hline Coated urea & - & - & $0.24 \mathrm{a}$ & $1.06 \mathrm{a}$ & $0.54 \mathrm{a}$ & $0.52 \mathrm{a}$ & $0.46 \mathrm{a}$ & $0.15 \mathrm{a}$ \\
\hline \multicolumn{9}{|c|}{ Average for fertilizer rate } \\
\hline- & 0 & - & $0.17 \mathrm{a}$ & $0.76 \mathrm{a}$ & $0.40 \mathrm{a}$ & $0.33 \mathrm{a}$ & $0.30 \mathrm{a}$ & $0.09 \mathrm{a}$ \\
\hline- & 90 & - & $0.28 \mathrm{~b}$ & $0.79 \mathrm{a}$ & $0.46 \mathrm{a}$ & $0.45 \mathrm{ab}$ & $0.37 \mathrm{a}$ & $0.14 \mathrm{~b}$ \\
\hline- & 180 & - & $0.37 \mathrm{c}$ & $1.39 \mathrm{~b}$ & $0.53 \mathrm{a}$ & $0.58 \mathrm{~b}$ & $0.54 \mathrm{~b}$ & $0.19 \mathrm{c}$ \\
\hline \multicolumn{9}{|c|}{ Average for dose dividing } \\
\hline- & - & $1 \times$ & $0.34 \mathrm{a}$ & $1.21 \mathrm{a}$ & $0.58 \mathrm{a}$ & $0.57 \mathrm{a}$ & $0.50 \mathrm{a}$ & $0.17 \mathrm{a}$ \\
\hline- & - & $2 \times$ & $0.28 \mathrm{a}$ & $0.96 \mathrm{a}$ & $0.42 \mathrm{a}$ & $0.46 \mathrm{a}$ & $0.43 \mathrm{a}$ & $0.15 \mathrm{a}$ \\
\hline \multicolumn{9}{|l|}{ Average for years } \\
\hline 2013 & & & $0.14 \mathrm{a}$ & $1.11 \mathrm{ab}$ & $0.82 \mathrm{~b}$ & $0.92 \mathrm{~b}$ & $0.78 \mathrm{~b}$ & $0.12 \mathrm{a}$ \\
\hline 2014 & & & $0.27 \mathrm{ab}$ & $1.39 \mathrm{~b}$ & $0.18 \mathrm{a}$ & $0.11 \mathrm{a}$ & $0.27 \mathrm{a}$ & $0.13 \mathrm{a}$ \\
\hline 2015 & & & $0.52 \mathrm{~b}$ & $0.76 \mathrm{a}$ & - & - & $0.35 \mathrm{a}$ & $0.23 \mathrm{~b}$ \\
\hline
\end{tabular}

Letters a and b: homogeneous groups according to the Duncan test, at $p<0.05$. The test was calculated separately for each month and independently for each factor (fertilizer type, fertilizer rate, dose dividing, year of experiment). The same letter indicates no difference between means for a particular factor in a particular month.

microorganisms, so it does not reach the shallow groundwater table. However, high concentrations of $\mathrm{N}-\mathrm{NO}_{3}$ in the subsoil layer, beyond the main rooting zone, may directly affect the quality of groundwater. The abovementioned threshold concentration of N$\mathrm{NO}_{3}, 11.3 \mathrm{mg} \mathrm{dm}{ }^{-3}$, was exceeded only in June and July (in both the topsoil and subsoil layers), while in the other months (including November), the $\mathrm{N}-\mathrm{NO}_{3}$ concentration was order of magnitude lower than the limit (Table 6). Thus, it may be concluded that the highest risk of nitrate leaching during the analysed period of June-November mainly occurred in the first part of the growing season, i.e. in June and July.

In the topsoil layer, a correlation was found between the $\mathrm{N}-\mathrm{NO}_{3}$ concentration in soil solutions and the kind of the applied nitrogen fertilizer (Table 2). For 4 months 


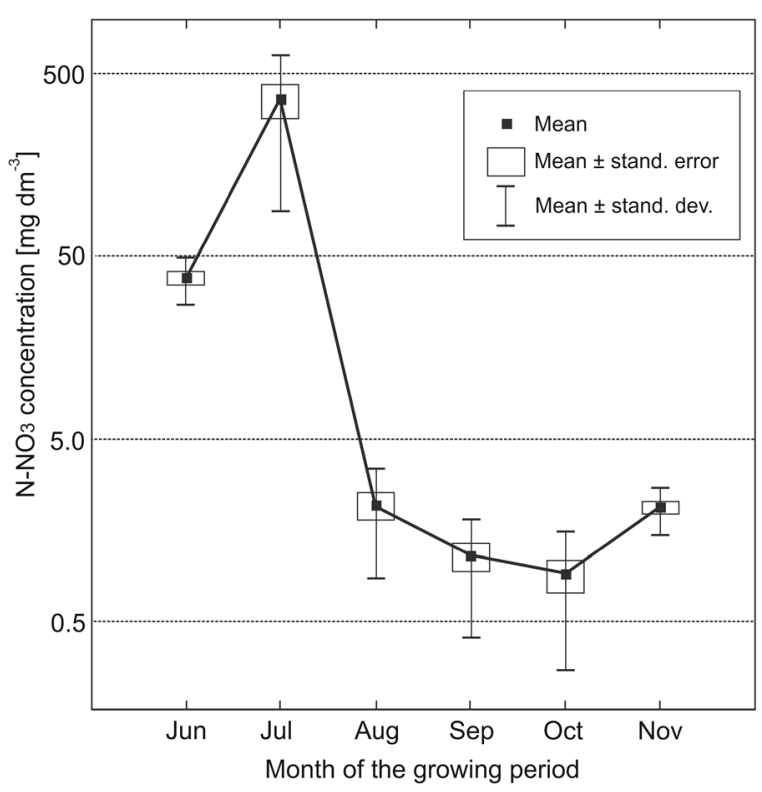

Fig. 1 Seasonal variability of $\mathrm{N}^{-\mathrm{NO}_{3}}$ concentration in the soil solution of topsoil layer (at the depth of $25 \mathrm{~cm}$ ). Month data averaged for the years 2013-2015

of the year (average for the years 2013-2015), the highest concentrations were noted for plots fertilized with standard urea (with an absolute maximum in July). On the contrary, the concentrations of $\mathrm{N}^{-\mathrm{NO}_{3}}$ in variants fertilized with slow-release urea were the lowest among the three analysed fertilizers in all the months of the vegetation season (the differences were statistically significant only for values in June and October).

The influence of fertilizer dose ( 90 or $180 \mathrm{~kg} \mathrm{ha}^{-1}$ ) on the $\mathrm{N}-\mathrm{NO}_{3}$ concentrations in soil solutions is also noticeable, as they were higher for the dose of 180 than for the dose of $90 \mathrm{~kg} \mathrm{ha}^{-1}$ (Table 2). The concentrations of
$\mathrm{N}-\mathrm{NO}_{3}$ on fertilized plots were significantly higher than on control plots, but the difference between fertilizer doses was statistically significant only in November.

Single or divided applications of nitrogen fertilizers resulted in the differentiation of $\mathrm{N}-\mathrm{NO}_{3}$ concentrations between the variants of the experiment. In line with expectations, the $\mathrm{N}-\mathrm{NO}_{3}$ concentrations were higher in June and July for a whole dose applied once at the beginning of the vegetation period (in July, this difference was highly statistically significant). Also, the higher $\mathrm{N}-\mathrm{NO}_{3}$ concentrations were noted in the period August-November for doses divided into two applications (the difference was significant only in September).

To sum up, the $\mathrm{N}^{-\mathrm{NO}_{3}}$ concentration in topsoil soil solution was influenced both by the type of fertilizer, fertilizer dose, and its division into parts. However, in the majority of the analysed monthly periods, the differences between specific variants of the experiments were insufficiently significant. This was, at least to a certain extent, due to the large differences between the results in subsequent years, in relation to weather conditions. Similar methodological problems have been pointed out, e.g. by Shaviv (2001), who emphasized that observation results from years colder or warmer than average or drier or more rainy than average may differ significantly, and this may affect the final conclusions. Although the trends noted in the field experiment with sorghum are insufficiently statistically significant, they are quite unambiguous and similar to results obtained by other authors with respect to the influence of the dose and division of doses of nitrogen fertilizers (Pietrzak and Sapek 1997) and the influence of slow-release fertilizers in comparison to their standard equivalents (Wilson et al. 2010).

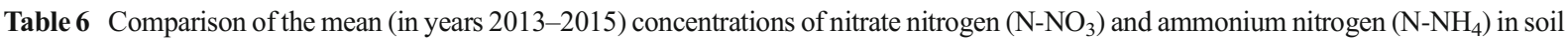
solution at the depth of $25 \mathrm{~cm}$ (topsoil) and $50 \mathrm{~cm}$ (subsoil)

\begin{tabular}{|c|c|c|c|c|}
\hline \multirow[t]{2}{*}{ Month } & \multicolumn{2}{|c|}{ Topsoil — depth $25 \mathrm{~cm}$} & \multicolumn{2}{|c|}{ Subsoil-depth $50 \mathrm{~cm}$} \\
\hline & $\mathrm{N}-\mathrm{NO}_{3}\left(\mathrm{mg} \mathrm{dm}^{-3}\right)$ & $\mathrm{N}-\mathrm{NH}_{4}\left(\mathrm{mg} \mathrm{dm}^{-3}\right)$ & $\mathrm{N}-\mathrm{NO}_{3}\left(\mathrm{mg} \mathrm{dm}^{-3}\right)$ & $\mathrm{N}-\mathrm{NH}_{4}\left(\mathrm{mg} \mathrm{dm}^{-3}\right)$ \\
\hline June & $38.3 \mathrm{~A} \mathrm{a}$ & $0.58 \mathrm{~B} \mathrm{a}$ & $22.2 \mathrm{~A} \mathrm{a}$ & $0.31 \mathrm{~B} \mathrm{a}$ \\
\hline July & $366 \mathrm{~A} \mathrm{a}$ & $1.47 \mathrm{~B} \mathrm{a}$ & $65.3 \mathrm{~A} \mathrm{~b}$ & $1.09 \mathrm{~B} \mathrm{a}$ \\
\hline August & $2.16 \mathrm{~A} \mathrm{a}$ & $1.46 \mathrm{~A} \mathrm{a}$ & $1.21 \mathrm{~A} \mathrm{~b}$ & $0.50 \mathrm{~B} \mathrm{~b}$ \\
\hline September & $1.13 \mathrm{~A} \mathrm{a}$ & $1.25 \mathrm{~A} \mathrm{a}$ & $0.48 \mathrm{~A} \mathrm{~b}$ & $0.52 \mathrm{~A} \mathrm{~b}$ \\
\hline October & $0.90 \mathrm{~A} \mathrm{a}$ & $0.56 \mathrm{~A} \mathrm{a}$ & $0.58 \mathrm{~A} \mathrm{a}$ & $0.47 \mathrm{~A} \mathrm{a}$ \\
\hline November & $2.15 \mathrm{~A} \mathrm{a}$ & $0.40 \mathrm{~B} \mathrm{a}$ & $0.73 \mathrm{~A} \mathrm{~b}$ & $0.16 \mathrm{~B} \mathrm{~b}$ \\
\hline
\end{tabular}

Homogeneous groups according to the Duncan test, at $p<0.05$ (the same letter indicates no difference between means for particular month): A, B- between $\mathrm{N}_{-} \mathrm{NO}_{3}$ and $\mathrm{N}-\mathrm{NH}_{4}$ in the same soil layer and a, b- between topsoil and subsoil (and separately for $\mathrm{N}-\mathrm{NO}_{3}$ and $\mathrm{N}-\mathrm{NH}_{4}$ ) 


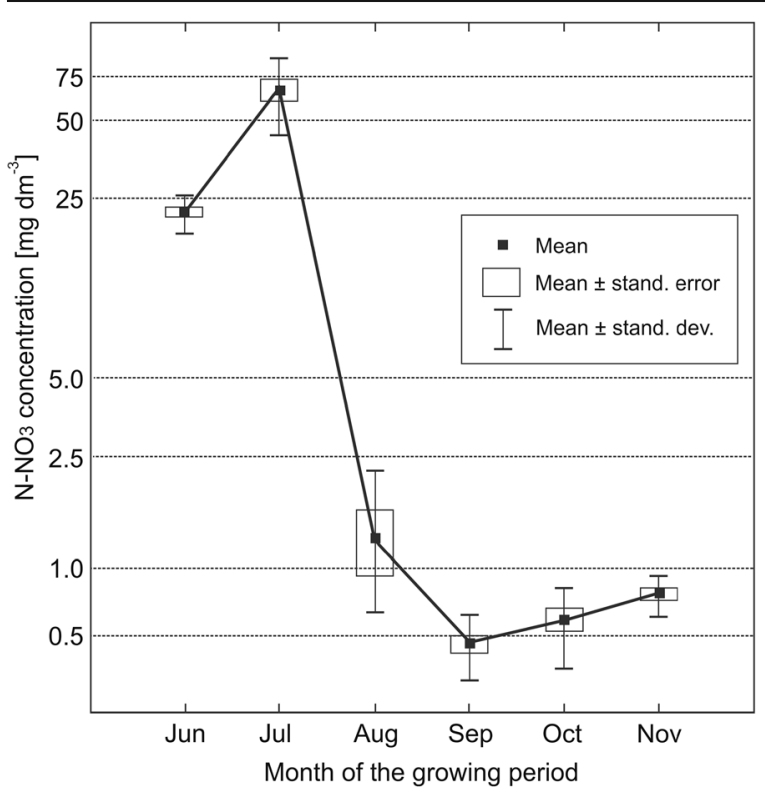

Fig. 2 Seasonal variability of $\mathrm{N}^{-\mathrm{NO}_{3}}$ concentration in the soil solution of subsoil layer (at the depth of $50 \mathrm{~cm}$ ). Month data averaged for the years 2013-2015

As expected, the concentration of $\mathrm{N}-\mathrm{NH}_{4}$ in soil solutions was significantly lower than that of $\mathrm{N}^{-\mathrm{NO}_{3}}$, but in summer (August-October), the mean concentrations became equal, or the concentration of $\mathrm{N}_{-} \mathrm{NH}_{4}$ was even slightly higher than that of $\mathrm{N}-\mathrm{NO}_{3}$ (Table 6). This phenomenon occurred in the topsoil (Fig. 3) and subsoil layers (Fig. 4). In late autumn (November), the concentration of $\mathrm{N}^{-\mathrm{NH}_{4}}$ in soils was again much lower than that of $\mathrm{N}-\mathrm{NO}_{3}$.

In annual terms, the range between minimum and maximum $\mathrm{N}-\mathrm{NH}_{4}$ concentrations (averaged for specific months) in the arable layer was approximately triple, i.e. considerably lower (narrower) than that for $\mathrm{N}^{-\mathrm{NO}_{3}}$ where highest values were nearly 400 times higher than the lowest ones (Table 6). The concentrations of $\mathrm{N}^{-\mathrm{NH}_{4}}$ in the subsoil layer were lower than in the topsoil layer, and ranged from $0.16 \mathrm{mg} \mathrm{dm}^{-3}$ (November) to $1.09 \mathrm{mg} \mathrm{dm}^{-3}$ (July). However, the differences between $\mathrm{N}-\mathrm{NH}_{4}$ concentrations in the topsoil and subsoil layers were statistically significant in late summer (August-September) only (Table 6).

The ranges of $\mathrm{N}_{-} \mathrm{NH}_{4}$ concentrations in soil solutions in the present experiment do not diverge from values provided by other authors, including Durkowski et al. (2007), who reported results from 0.1 to $1.8 \mathrm{mg} \mathrm{N}$ $\mathrm{NH}_{4} \mathrm{dm}^{-3}$ in the soils of the Pyrzycka Lowland.

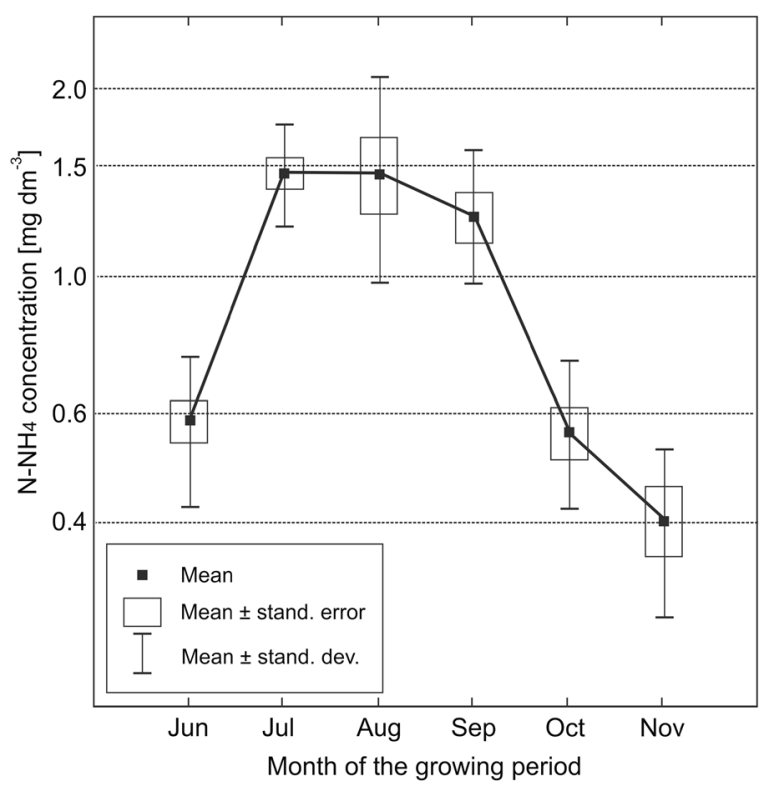

Fig. 3 Seasonal variability of $\mathrm{N}-\mathrm{NH}_{4}$ concentration in the soil solution of topsoil layer (at the depth of $25 \mathrm{~cm}$ ). Month data averaged for the years 2013-2015

The influence of the type of nitrogen fertilizer on the concentration of $\mathrm{N}_{-} \mathrm{NH}_{4}$ in the topsoil soil solution is not clear; however, the highest concentrations were generally noted for standard urea fertilization (Table 3). The highest $\mathrm{N}-\mathrm{NH}_{4}$ concentration in the subsoil was also found in the variant fertilized with standard urea, but

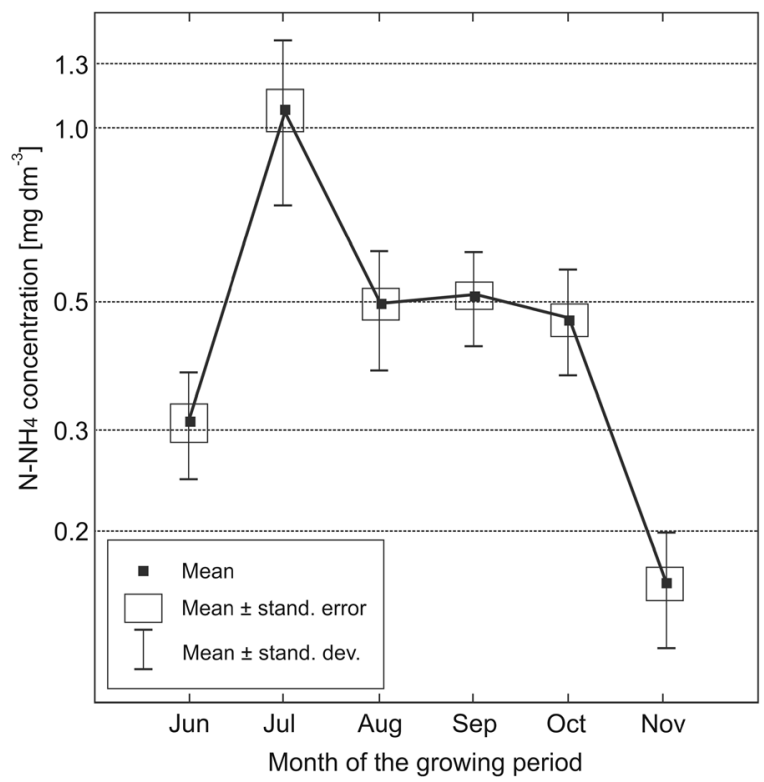

Fig. 4 Seasonal variability of $\mathrm{N}-\mathrm{NH}_{4}$ concentration in the soil solution of subsoil layer (at the depth of $50 \mathrm{~cm}$ ). Month data averaged for the years 2013-2015 
the differences between fertilizers were very little and statistically insignificant, with the exception of June, when significantly lower concentrations of $\mathrm{N}^{-\mathrm{NH}_{4}}$ were noted in the variant with slow-release (coated) urea (Table 5).

Higher doses of nitrogen fertilizers resulted in elevated concentrations of $\mathrm{N}-\mathrm{NH}_{4}$ in topsoil soil solutions, but the differences were statistically insignificant, except for June (Table 3). Also the concentration of $\mathrm{N}^{-\mathrm{NH}_{4}}$ in the subsoil was considerably higher in the variant with the $90 \mathrm{~kg} \mathrm{ha}^{-1}$ dose than with the $90 \mathrm{~kg} \mathrm{ha}^{-1}$ during a major part of the vegetation season (Table 5).

In previous studies, ammonium ions had been considered less mobile than the nitrate ions, or more prone to transformation, which supposedly led to a reduction of their concentrations in soil solutions. Thus, $\mathrm{N}-\mathrm{NH}_{4}$ was not considered to be a serious threat for the quality of groundwater (Jadczyszyn et al. 2010). The only reference that can be applied for the concentrations of $\mathrm{N}$ $\mathrm{NH}_{4}$ in soil solutions is the threshold value specified in the regulation for groundwaters (Regulation 2008), i.e. $1.24 \mathrm{mg} \mathrm{N}_{-} \mathrm{NH}_{4} \mathrm{dm}^{-3}\left(1.5 \mathrm{mg} \mathrm{NH}_{4} \mathrm{dm}^{-3}\right)$ respective for "satisfactory" water quality (class III). In the topsoil layer, this value was exceeded in June and July, both averaged for the whole test period (Fig. 3) and in numerous individual variants (Table 3 ). In the subsoil layer, it was exceeded in June in variants fertilized with the $180 \mathrm{~kg} \mathrm{ha}^{-1}$ dose only. Thus, it should be concluded that the quality of shallow groundwater is threatened by ammonium nitrate from fertilizers, but only at a high dose (at least $180 \mathrm{~kg} \mathrm{ha}^{-1}$ ) of nitrogen fertilization.

\section{Conclusions}

1. Miniaturized composite suction cups MacroRhizon enable the precise monitoring of the concentration of nitrate and ammonium ions under field conditions, at various soil depths and in a large number of replications. The main factors limiting their usability are sandy texture of soil and seasonal water deficit in capillary pores related to summer droughts.

2. Both the ammonium and nitrate ions reached their maximum concentrations in the soil solutions during the growing season in June-July and the minimum concentrations in September-October. The maximum concentrations of $\mathrm{N}-\mathrm{NH}_{4}$ were over ten times higher than the minimum ones, whereas the maximum concentrations of $\mathrm{N}-\mathrm{NO}_{3}$ were up to 400 times higher than the minimum ones during the growing season.

3. The highest concentrations of ammonium and nitrate ions in the soil solution were in the variant fertilized with standard urea, followed by ammonium nitrate, and the slow-release (coated) urea. The dose of $180 \mathrm{~kg} \mathrm{ha}^{-1}$ of nitrogen fertilizers resulted in a higher concentration of nitrate and ammonium ions in soil solutions throughout the growing period compared to the dose of $90 \mathrm{~kg} \mathrm{ha}^{-1}$, in both the topsoil and subsoil layers. Single application of the entire fertilizer dose resulted in a higher concentration of $\mathrm{N}-\mathrm{NH}_{4}$ and $\mathrm{N}-\mathrm{NO}_{3}$ in the subsoil soil solution throughout the entire growing season, compared to divided dose. In the topsoil soil solution, single application of the fertilizer led to a higher concentration of $\mathrm{N}-\mathrm{NH}_{4}$ and $\mathrm{N}-\mathrm{NO}_{3}$ in June-July, whereas dividing the dose raised their concentrations in the period August-November.

4. In the subsoil layer, the concentration of $\mathrm{N}_{-} \mathrm{NO}_{3}$ in soil solution exceeded the reference values as for groundwater in June and July (for both doses of nitrogen fertilizers), and the concentrations of $\mathrm{N}$ $\mathrm{NH}_{4}$ exceeded the threshold in July (only for high dose of fertilization), which indicates the potential threat of nitrogen leaching (during the growing season) in June and July, mainly related to the nitrate forms.

Acknowledgements The research was financed by the Polish National Centre for Research and Development (NCBiR) as a part of a project "Agrotechnical, environmental, and technological aspects of nitrogen fertilization of sweet sorghum", grant No. PBS1/A8/11/2013.

Open Access This article is distributed under the terms of the Creative Commons Attribution 4.0 International License (http:// creativecommons.org/licenses/by/4.0/), which permits unrestricted use, distribution, and reproduction in any medium, provided you give appropriate credit to the original author(s) and the source, provide a link to the Creative Commons license, and indicate if changes were made.

\section{References}

Berge, H., Fotyrnaz, E., Cermak, P., Bujnovski, R., Karklins, A., Poguliss, A., \& Bos, J. (2001). Using crop nitrogen response functions in fertilizer recommendation at field and farm level. Nawozy i Nawożenie, 2(7), 77-94. 
Bouwman, A. F., Bierkens, M. F. P., Griffioen, J., Hefting, M. M., Middelburg, J. J., Middelkoop, H., \& Slomp, C. P. (2013). Nutrient dynamics, transfer and retention along the aquatic continuum from land to ocean: towards integration of ecological and biogeochemical models. Biogeosciences, 10(1), $1-22$.

Csathó, P., Sisák, I., Radimszky, L., Lushaj, S., Spiegel, H., Nikolova, M. T., \& Karklins, A. (2007). Agriculture as a source of phosphorus causing eutrophication in Central and Eastern Europe. Soil Use and Management, 23(s1), 36-56.

Cuske, M., Karczewska, A., \& Gałka, B. (2017). Speciation of $\mathrm{Cu}$, $\mathrm{Zn}$, and $\mathrm{Pb}$ in soil solutions extracted from strongly polluted soils treated with organic materials. Polish Journal of Environmental Studies, 26, 567-575.

Dojlido, J., \& Zerbe, J. (1997). Instrumental methods of water and wastewater analysis. Warszawa: Arkady $274 \mathrm{pp}$.

Duer, I., Fotyma, M., \& Madej, A. (2002). Codex of good agricultural practice. Warszawa: Ministry of Agriculture, Ministry of Environment 112 pp.

Durkowski, T., Burczyk, P., \& Królak, B. (2007). Concentrations of selected chemical components in ground waters and soil solution of a small agricultural catchment basin. Woda Środowisko - Obszary Wiejskie, 7, 5-15.

Fotyma, M., Kęsik, K., \& Pietruch, C. (2010). Mineral nitrogen in soils of Poland as an indicator of plants nutrient requirements and soil water cleanness. Nawozy i Nawożenie, 38, 5-83.

Gabriel, J. L., Muñoz-Carpena, R., \& Quemada, M. (2012). The role of cover crops in irrigated systems: water balance, nitrate leaching and soil mineral nitrogen accumulation. Agriculture Ecosystems and Environment, 155, 50-61.

Gałka, B., Kabała, C., Karczewska, A., Sowiński, J., \& Jakubiec, J. (2016). Variability of soil properties in an intensively cultivated experimental field. Soil Science Annual, 67(1), 10-16.

Georgallas, A., Dessureault-Rompré, J., Zebarth, B. J., Burton, D. L., Drury, C. F., \& Grant, C. A. (2012). Modification of the biophysical water function to predict the change in soil mineral nitrogen concentration resulting from concurrent mineralization and denitrification. Canadian Journal of Soil Science, 92(5), 695-710.

Glina, B., Bogacz, A., \& Woźniczka, P. (2016). Nitrogen mineralization in forestry-drained peatland soils in the Stołowe Mountains National Park (Central Sudetes Mts). Soil Science Annual, 67(2), 64-72.

Hatch, D. J., Joynes, A., \& Stone, A. (2010). Nitrogen uptake in organically managed spring sown lupins and residual effects on leaching and yield of a following winter cereal. Soil Use and Management, 26(1), 21-26.

Iost, S., Rautio, P., \& Lindroos, A. J. (2012). Spatio-temporal trends in soil solution $\mathrm{BC} / \mathrm{Al}$ and $\mathrm{N}$ in relation to critical limits in European forest soils. Water Air Soil Pollution, 223, 1467-1479.

Jadczyszyn, T., Pietruch, C., \& Lipiński, W. (2010). Soil monitoring in Poland for the content of mineral nitrogen in the years 2007-2009. Fertizers and Fertilization, 38, 84-110.

Jämtgård, S., Näsholm, T., \& Huss-Danell, K. (2010). Nitrogen compounds in soil solutions of agricultural land. Soil Biology and Biochemistry, 42(12), 2325-2330.

Kabala, C., Karczewska, A., \& Medyńska-Juraszek, A. (2014). Variability and relationships between $\mathrm{Pb}, \mathrm{Cu}$, and $\mathrm{Zn}$ concentrations in soil solutions and forest floor leachates at heavily polluted sites. Journal of Plant Nutrition and Soil Science, 177, 573-584.

Kabala, C., Musztyfaga, E., Gałka, B., Łabuńska, D., \& Mańczyńska, P. (2016a). Conversion of soil pH 1:2.5 KCl and $1: 2.5 \mathrm{H} 2 \mathrm{O}$ to $1: 5 \mathrm{H} 2 \mathrm{O}$ - conclusions for soil management, environmental monitoring and international soil databases. Polish Journal of Environmental Studies, 25(3), 647653.

Kabala, C., Świtoniak, M., \& Charzyński, P. (2016b). Correlation between the Polish Soil Classification (2011) and international soil classification system World Reference Base for Soil Resources (2015). Soil Science Annual, 67(2), 88-100.

Kroes, J., \& Roelsma, J. (2007). Simulation of water and nitrogen flows on field scale; application of the SWAP-ANIMO model for the Müncheberg data set. In Modelling water and nutrient dynamics in soil-crop systems. Netherlands: Springer.

Labaz, B., \& Kabala, C. (2016). Human-induced development of mollic and umbric horizons in drained and farmed swampy alluvial soils. Catena, 139, 117-126.

Meisinger, J. J., \& Delgado, J. A. (2002). Principles for managing nitrogen leaching. Journal of Soil and Water Conservation, 57(6), 485-498.

Perego, A., Basile, A., Bonfante, A., De Mascellis, R., Terribile, F., Brenna, S., \& Acutis, M. (2012). Nitrate leaching under maize cropping systems in Po Valley (Italy). Agriculture, Ecosystems \& Environment, 147, 57-65.

Pietrzak, S., \& Sapek, B. (1997). The influence of soil pH and the form of nitrogen fertilizer on the content of nitrate-nitrogen and ammonium-nitrogen in soil solutions. Journal of Water and Land Development, 1, 83-91.

Regulation. (2002). Regulation of Ministry of Environment (Poland) on criteria for indication of waters sensitive to contamination with nitrogen compounds from agricultural sources. Dz.U. 2002.241.2093.

Regulation. (2008). Regulation of Ministry of Environment (Poland) on criteria and methods of groundwater quality assessment. Dz.U. 2008.143.896.

Sapek, A. (2004). Agricultural activities as a source of nitrates in groundwater. In L. Razowska-Jaworek \& A. Sadurski (Eds.), Nitrates in groundwater: IAH selected papers on hydrogeology (pp. 3-14). Leiden: Balkema Publ.

Sapek, B. (2010). Nitrogen and phosphorus release from soil organic matter. Woda - Środowisko - Obszary Wiejskie, 10, 229-256.

Shaviv, A. (2001). Advances in controlled-release fertilizers. Advances in Agronomy, 71, 1-49.

Siepak, J. (1992). Physico-chemical analysis of waters and soils. Poznań: Wydawnictwo UAM 225 pp.

Sierra, C. A., Trumbore, S. E., Davidson, E. A., Vicca, S., \& Janssens, I. (2015). Sensitivity of decomposition rates of soil organic matter with respect to simultaneous changes in temperature and moisture. Journal of Advances in Modeling Earth Systems, 7, 335-356.

Sowiński, J., Kabała, C., Karczewska, A., Szydełko-Rabska, E., \& Gałka, B. (2016). Content of mineral nitrogen in sandy soils after an application of slow-release fertilisers in sweet sorghum cultivation. Journal of Elementology, 21(4), 11271139.

Spohn, M., Novák, T. J., Incze, J., \& Giani, L. (2016). Dynamics of soil carbon, nitrogen, and phosphorus in calcareous soils 
after land-use abandonment - a chronosequence study. Plant and Soil, 401(1-2), 185-196.

Trindade, H., Coutinho, J., Van Beusichem, M. L., Scholefield, D., \& Moreira, N. (1997). Nitrate leaching from sandy loam soils under a double-cropping forage system estimated from suction-probe measurements. Plant and Soil, 195(2), 247256.

Webster, C. P., Shepherd, M. A., Goulding, K. W. T., \& Lord, E. (1993). Comparisons of methods for measuring the leaching of mineral nitrogen from arable land. Journal of Soil Science, 44(1), 49-62.
Wilson, M. L., Rosen, C. J., \& Moncrief, J. F. (2010). Effects of polymer-coated urea on nitrate leaching and nitrogen uptake by potato. Journal of Environmental Quality, 39(2), 492499.

Zakarauskaite, D., Vaisvila, Z., Motuzas, A., Grigaliuniene, K., Buivydaite, V. V., Vaisvalavicius, R., \& Butkus, V. (2008). The influence of long-term application of mineral fertilizers on the biological activity of Cambisols. Ekologija, 54(3), 173-178. 